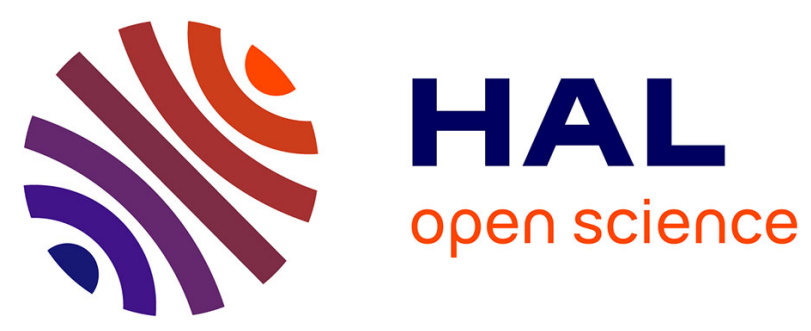

\title{
Simultaneous Tissue and Flow Estimation at High Frame Rate Using Plane Wave and Transverse Oscillation on in Vivo Carotid
}

\author{
Vincent Perrot, Hervé Liebgott, Anne Long, Didier Vray
}

\section{- To cite this version:}

Vincent Perrot, Hervé Liebgott, Anne Long, Didier Vray. Simultaneous Tissue and Flow Estimation at High Frame Rate Using Plane Wave and Transverse Oscillation on in Vivo Carotid. 2018 IEEE International Ultrasonics Symposium (IUS), Oct 2018, Kobe, Japan. pp.1-4, 10.1109/ULTSYM.2018.8580182 . hal-02063521

\section{HAL Id: hal-02063521 \\ https://hal.science/hal-02063521}

Submitted on 11 Mar 2019

HAL is a multi-disciplinary open access archive for the deposit and dissemination of scientific research documents, whether they are published or not. The documents may come from teaching and research institutions in France or abroad, or from public or private research centers.
L'archive ouverte pluridisciplinaire HAL, est destinée au dépôt et à la diffusion de documents scientifiques de niveau recherche, publiés ou non, émanant des établissements d'enseignement et de recherche français ou étrangers, des laboratoires publics ou privés. 


\title{
Simultaneous Tissue and Flow Estimation at High Frame Rate using Plane Wave and Transverse Oscillation on In Vivo Carotid
}

\author{
Vincent Perrot*, Hervé Liebgott*, Anne Long*†, Didier Vray* \\ *Univ. Lyon, INSA-Lyon, UCBL, UJM-Saint-Étienne, CNRS, Inserm, CREATIS UMR 5220, U1206, Lyon, France \\ vincent.perrot@creatis.insa-lyon.fr \\ ${ }^{\dagger}$ Department of Internal Medicine and Vascular Medicine, Hospices Civils de Lyon, Hôpital Edouard Herriot, Lyon, France
}

\begin{abstract}
In this study, a method to estimate tissue motion and vector flow fields, from a unique ultrasound plane wave sequence, was applied to several in vivo carotid data sets. Moreover, pulse wave velocity was also extracted thanks to the wall velocity estimates. Acquisitions were performed at high frame rate using horizontal plane waves without compounding $(5000 \mathrm{~Hz})$. Results showed that the method could simultaneously yield wall velocity and flow in case of low or high flow rates and even when complex patterns occur. Moreover, pulse wave velocities were estimated from two distinct waves; one is measured from the foot of displacement (forward wave) while the second one is extracted at the dicrotic notch. The forward wave has been estimated with a value of $3.25 \pm 0.81 \mathrm{~m} / \mathrm{s}$ while the second one has been measured at $5.43 \pm 1.30 \mathrm{~m} / \mathrm{s}$; the difference can be explained with the two different pre-loads on the artery wall. Overall, the method can simultaneously extract fast and complex phenomena that can occur in both tissue and flow. Thanks to the simultaneous assessment of those characteristics, new pathological indicators could be discovered by studying the relationship and synchronization between flow and wall motion.
\end{abstract}

Index Terms-Tissue motion, flow, pulse wave, high frame rate, in vivo, carotid, transverse oscillation

\section{INTRODUCTION}

Modifications of artery properties can be related to natural causes such as aging [1], [2] or pathological causes such as atherosclerosis, hypertension or diabetes [3], [4]. These modifications influence the flow pattern and the wall motion due to changes in its elasticity which can be assessed through the measurement of the pulse wave velocity (PWV). PWV has been proved to be an efficient indicator of cardiovascular risk [5]. Consequently, precise evaluation of these characteristics should be highly relevant for the detection and characterization of pathologies during their development or even at the earliest stage.

For the last twenty years, both technological and scientific

The Verasonics system was co-funded by the FEDER program, Saint-Etienne Metropole (SME) and Conseil General de la Loire (CG42) within the SonoCardioProtection Project headed by Prof. Pierre Croisille and Dr. Magalie Viallon, principal investigators. This work has been performed within the framework of the LABEX CELYA (ANR-10-LABX-0060) and LABEX PRIMES (ANR-10-LABX-0063) of Universite de Lyon, within the program "Investissements d'Avenir" (ANR-11-IDEX-0007) operated by the French National Research Agency (ANR). advances have allowed the emergence of new ultrasound imaging sequences at high frame rate using plane or diverging waves [6]. Thanks to these advancements, ultrasound sequences can now be developed with a high temporal resolution which permits to imagine complex and fast phenomena of the artery wall and flow. In parallel, several studies have focused on the assessment of flow velocity [7] and wall motion for PWV estimation [8] but separately. All these studies have shown the potential of ultrafast ultrasound sequences by providing characteristics of the cardiovascular system that cannot be assessed at lower frame rates. However, the simultaneous measurement of flow, wall motion as well as PWV would be highly valuable for clinicians to detect or evaluate the severity level of pathologies by linking wall and flow characteristics.

Several groups developed various technics and ultrasound schemes to simultaneously assess characteristics of flow and wall artery at high frame rate. Hasegawa and Kanai [9] were among the very first by providing a phase-tracking method for assessing the radial strain of the wall combined with a power Doppler mode for the flow. Later, Luo and Konofagou [10] have conducted a feasibility study of imaging cardiovascular wall motion coupled with blood flow on in vivo heart mice using speckle tracking. More recently, Ekroll et al. [11] have evaluated a quantitative angle-independent $2 \mathrm{D}$ vector velocity estimator for both wall motion and flow assessment evaluated on realistic carotid simulations and in vivo carotids. Although improvements were made for the simultaneous assessment of blood and wall motion properties, none of those previous studies have extracted PWV with simultaneous measurement of flow and wall motion. In the following, a method using a phase-based estimator on radio-frequency (RF) signals has been applied on in vivo carotid data sets at high frame rate for wall motion and flow estimation. Afterward, PWV has been extracted based on the estimates. This method was validated previously with an experimental phantom set-up [12].

The section II introduces the ultrasound sequences and the methods used to estimate the wall motion, flow, and PWV. The section III presents the results. Finally, the section IV concludes the study with a summary and discusses the possible applications of the presented approach. 


\section{MATERIAL AND METHODS}

\section{A. Acquisition}

In this study, a Verasonics ultrasound system (Verasonics Inc., Redmond, WA) with the L7-4 probe were used. The acquisition parameters are described in Table I and data were beamformed using a conventional delay-and-sum (DAS) algorithm with an f-number of 1.5 .

All the acquisitions were performed on healthy volunteers, six subjects under the age of thirty who stood at rest for 20 minutes before the acquisitions. These requirements ensure that all phenomena which occur during acquisition are entirely natural and not due to an external cause.

TABLE I

ACQUISITION PARAMETERS

\begin{tabular}{ll}
\hline \hline Parameter & Value \\
\hline Pitch & $298 \mu \mathrm{m}$ \\
Number of elements & 128 \\
Transmit frequency & $5 \mathrm{MHz}$ \\
Sampling frequency & $20 \mathrm{MHz}$ \\
Transmit pulse & 3 -cycle sinusoidal pulse \\
Speed of sound & $1540 \mathrm{~m} / \mathrm{s}$ \\
Pulse repetition frequency & $5000 \mathrm{~Hz}$ \\
Compounding & No \\
Steering angle & $0^{\circ}$ (unsteered) \\
Transmit apodization & Tukey $50 \%$ \\
Receive apodization & Rectangular \\
Duration & $2 \mathrm{~s}$ \\
\hline \hline
\end{tabular}

\section{B. Velocity estimation}

The first step is the wall motion estimation; axial wall velocity is estimated using the complex autocorrelation function [13], [14] after I/Q demodulation at the transmit frequency

$$
v_{z}=-\frac{c \times P R F}{4 \pi f_{0}} \times \frac{\angle \hat{R}(1)}{\pi}
$$

where $c$ is the speed of sound $(\mathrm{m} / \mathrm{s}), P R F$ stands for the pulse repetition frequency $(\mathrm{Hz}), f_{0}$ corresponds to the transmit frequency $(\mathrm{Hz})$ and $\hat{R}(1)$ is the complex autocorrelation function at lag 1. A spatial average with a 2D Hanning kernel (size: $x=2.5 \mathrm{~mm}, z=1 \mathrm{~mm}$ ) is applied to the complex autocorrelation function with an ensemble length of 16 frames to further improve the estimates.

Afterward, a temporal adaptive FIR clutter filter (order 200) with a temporal adaptive cutoff (maximum of the axial wall velocity estimated in the current frame) is applied to the beamformed data to separate flow from tissue signal. The frequency responses of the FIR filters are shown in Fig. 1.

Finally, for the flow estimation, transverse oscillation (TO) was introduced in post-acquisition in the Fourier domain [15] in clutter filtered signals; this method consists to multiply the Fourier spectrum of each RF image with a Gaussian mask to only keep the desired lateral wavelength. The resulting Fourier spectrum is composed of four spots corresponding to the axial (natural) and lateral (introduced with the mask multiplication) frequencies. Afterward, 2D analytic signals

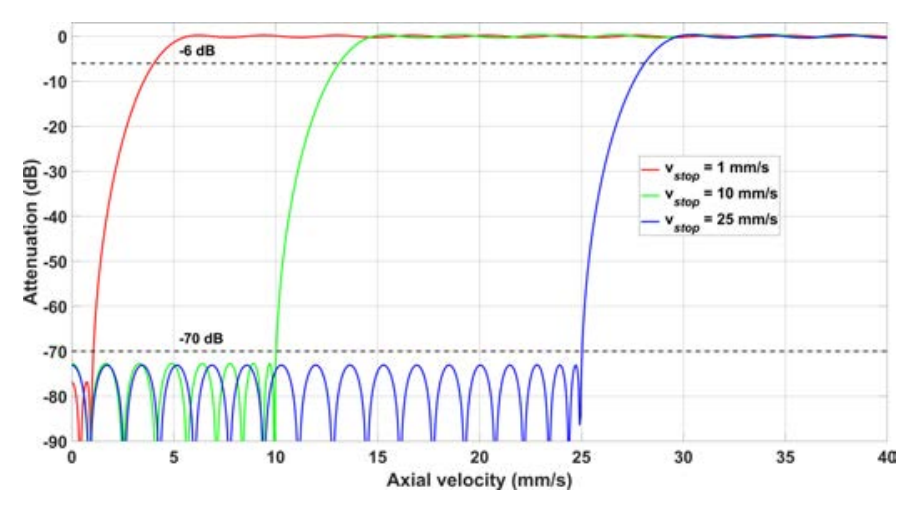

Fig. 1. Three impulse responses of the temporal adaptive FIR filters used for three cutoff: $1 \mathrm{~mm} / \mathrm{s}$ (minimum), $10 \mathrm{~mm} / \mathrm{s}$ (medium) and $25 \mathrm{~mm} / \mathrm{s}$ (maximum).

were extracted in the Fourier domain by selecting two different single quadrants [16]. Phase shifts were estimated using the 2D analytics signals

$$
\left\{\begin{array}{l}
\Phi_{1}=\angle \hat{R}_{1}(1) \\
\Phi_{2}=\angle \hat{R}_{2}(1)
\end{array}\right.
$$

where $\hat{R}_{1}(1)$ denotes the complex autocorrelation function of the first quadrant analytic signal at lag 1 and $\hat{R}_{2}(1)$ is the same function but for the second quadrant analytic signal. Motion is recovered by projecting the phase shift onto the axial and lateral axes

$$
\left\{\begin{array}{l}
v_{z}=-\frac{c \times P R F}{4 \pi f_{0}} \times \frac{\Phi_{1}+\Phi 2}{\pi} \\
v_{x}=-\frac{c \times P R F}{4 \pi f_{x}} \times \frac{\Phi_{1}-\Phi 2}{\pi}
\end{array}\right.
$$

where $f_{x}\left(\mathrm{~m}^{-1}\right)$ is the lateral frequency of TO. A spatial average with a $2 \mathrm{D}$ rectangular kernel (size: $x=2.5 \mathrm{~mm}$, $z=1 \mathrm{~mm}$ ) is applied to the complex autocorrelation function with an ensemble length of 64 frames to further improve the estimates.

\section{Pulse wave velocity estimation}

After wall motion estimation, the pulse wave can be identified thanks to wall acceleration; a gradient is applied on wall velocity along time to compute the acceleration. The instant where the pulse wave passes through the wall is described by an acceleration peak along the time axis. Moreover, two pulse waves can be identified [17] during the cardiac cycle as showed for one position along the proximal wall in Fig. 2.

Both waves are extracted thanks to the acceleration (red curve in Fig. 2b) obtained from the velocity estimate (red curve in Fig. 2a). The first wave occurs at the foot of motion (forward wave) while the second wave occurs during the dicrotic notch. Because these two waves occur at two different times and the diameter of the artery is different, the velocities are different due to two different pre-loads on the arterial wall tissue in case of healthy and also diseased carotid tissue [18]. To estimate the 


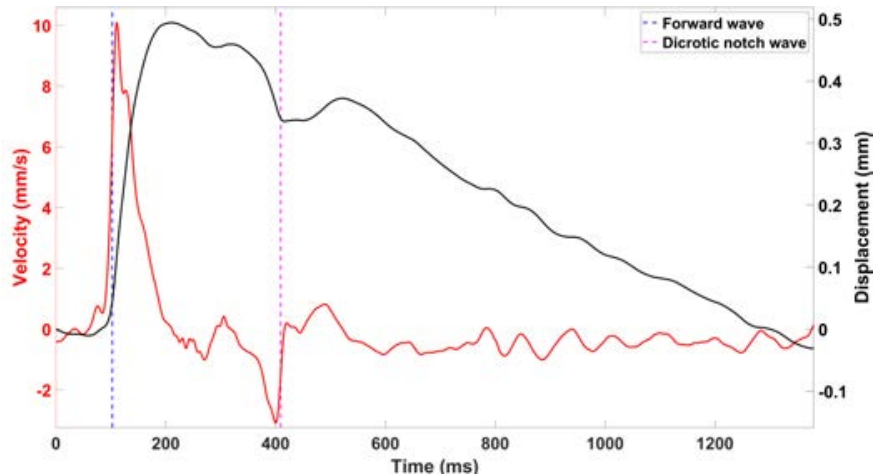

(a)

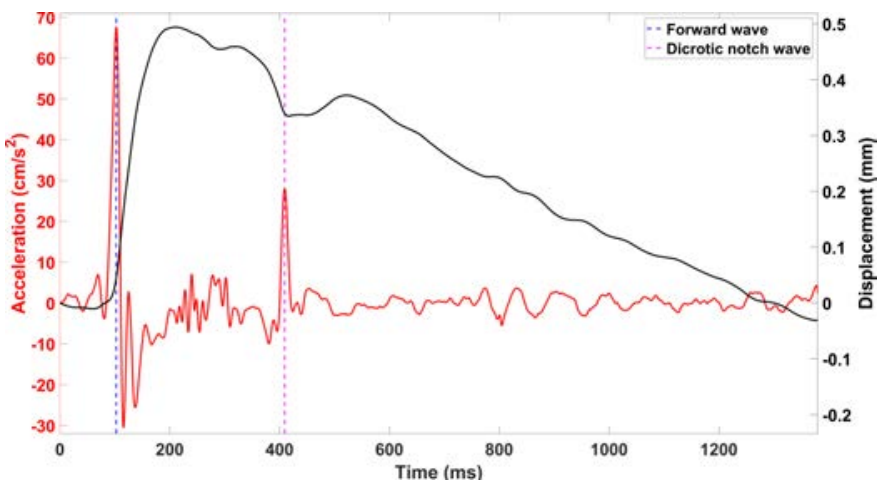

(b)

Fig. 2. Wall velocity and displacement (a) for the center of the proximal wall during one cardiac cycle. Two waves are detected (dotted lines) based on the wall acceleration (b).

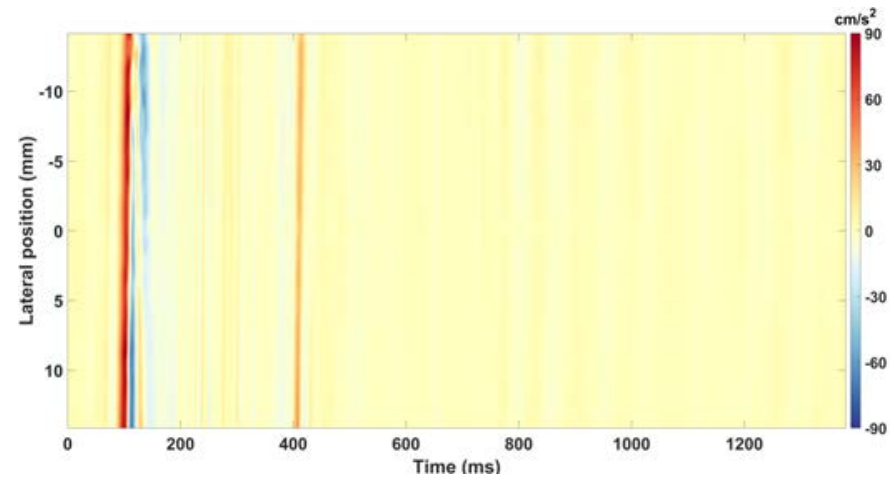

Fig. 3. Acceleration map of the proximal wall where two pulse waves can be extracted at $100 \mathrm{~ms}$ (forward wave) and $410 \mathrm{~ms}$ (dicrotic notch wave).

PWV the full acceleration map is computed for each position along the wall (Fig. 3). The PWV is estimated based on the local maximums (Fig. 4) of the acceleration map. A linear regression is applied to the local maximums, which occur at different times along the wall due to the propagation, to compute the pulse wave velocity.

\section{RESULTS}

\section{A. Flow and wall velocity}

One of the interests of this method is to visualize flow and wall motion simultaneously. An example of this kind of

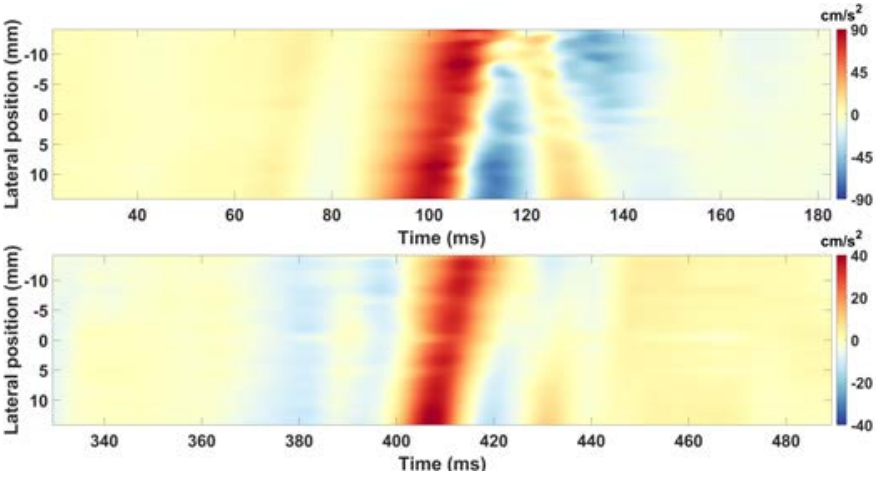

Fig. 4. Close-up views of the acceleration map of the proximal wall for the two pulse waves at $100 \mathrm{~ms}$ (top, forward wave) and $410 \mathrm{~ms}$ (bottom, dicrotic notch wave).

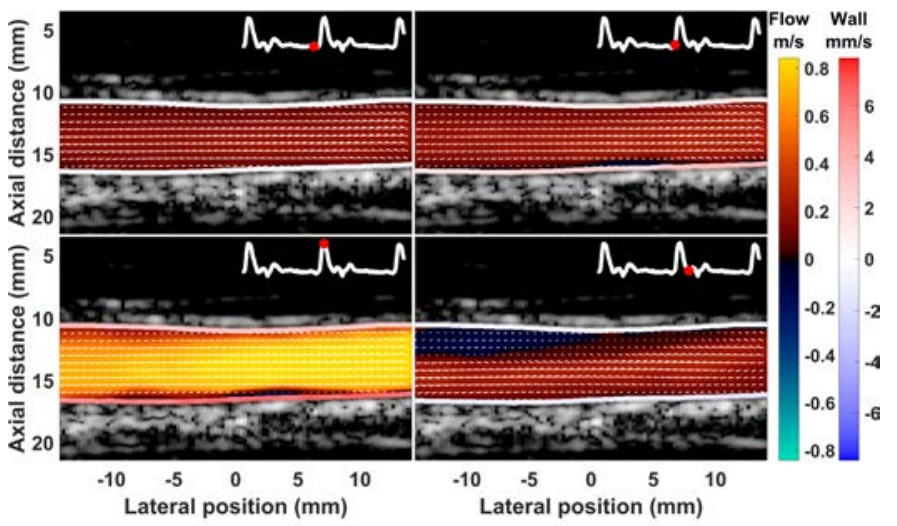

Fig. 5. Four snapshots of the B-mode images superimposed with the flow and the wall velocity at four different times: before systole (top-left), when the pulse wave arrives from the right (top-right), at the systolic peak (bottom-left) and just after the systole (bottom-right). The heart is located on the right while brain is on the left. A positive value for the wall velocity stands for an expansion while a negative value means a contraction of the vessel. The white curve indicates the flow at the center of the lumen across time.

visualization is provided (Fig. 5) for one acquisition.

The visualization shows that the method extracts the flow even with complex pattern (Fig. 5, bottom-right); indeed, a vortex due to the proximity of the carotid bulb two centimeters away from the left part of the B-mode images can be visualized. Low (Fig. 5, top-left) and high (Fig. 5, bottom-left) flow velocities can also be estimated. Moreover, the method provides simultaneous visualization of flow and tissue motion.

\section{B. Pulse wave velocity}

During this study, three acquisitions of two seconds each were performed for each volunteer. Depending on the duration of the cardiac cycles which varies between volunteers and the position in the cardiac cycle at the beginning of each acquisition (not synchronized with ECG) 1 to 3 full cardiac cycles can be analyzed per acquisition. In total, 58 pulse waves were detected, and the resulting estimated velocities (PWV) are summarized in Table II with the standard deviation and the coefficient of determination $\left(\mathrm{r}^{2}\right)$ of the linear regression. The dicrotic notch wave has a higher value than the forward 
wave. The pre-load can explain this difference due to the displacement of the wall. Indeed, the diameter of the vessel is larger at the dicrotic notch, which occurs at the end of the systole, while the forward wave propagates at the very beginning of the systole.

TABLE II

ESTIMATED PULSE WAVE VELOCITIES

\begin{tabular}{|c|c|c|}
\hline Wave & PWV $(\mathrm{m} / \mathrm{s})$ & $\mathrm{r}^{2}$ \\
\hline Forward & $3.25 \pm 0.81(25 \%)$ & 0.861 \\
Dicrotic notch & $5.43 \pm 1.30(24 \%)$ & 0.939 \\
\hline
\end{tabular}

\section{CONCLUSION AND DISCUSSION}

In this study, an imaging method able to simultaneously provide flow, and PWV extracted from wall motion estimates has been applied on in vivo carotid data sets from young healthy volunteers.

The results show a complex flow pattern, low and high flow rate, and laminar flow. Moreover, two different waves have been estimated from the foot of displacement and the dicrotic notch with a value of $3.25 \pm 0.81 \mathrm{~m} / \mathrm{s}$ for the first wave and $5.43 \pm 1.30 \mathrm{~m} / \mathrm{s}$ for the last one. Overall, the method can describe fast and complex phenomena which occur in both tissue and flow on in vivo carotid with plane waves at high frame rate.

All these information extracted simultaneously may bring to the clinicians a useful tool by providing flow and wall characteristics in one acquisition. Moreover, because only one acquisition is performed, new characteristics can be studied as the delay between the two different waves corresponding to the atrial systole (foot of displacement) and the aortic valve closure (dicrotic notch). It also becomes possible to study the relationship between wall and flow which are inherently linked. Indeed, due to the incompressible nature of the blood, the flow should be triggered by the wall motion near the wall. One other application could be the measurement of the time shift between the flow and wall velocity peaks. Finally, because the method yields a local measurement of the pulse wave velocity as a result of the arterial wall stiffness and simultaneously the blood flow in particular, close to atherosclerotic lesions, it may contribute to assessing the vulnerability of the plaque. As a result, to further explore the clinical potential of the simultaneous pulse wave/flow imaging method proposed, future work will include pathological carotid data sets.

\section{REFERENCES}

[1] A. P. Avolio, S. G. Chen, R. P. Wang, C. L. Zhang, M. F. Li, and M. F. O'Rourke, "Effects of aging on changing arterial compliance and left ventricular load in a northern chinese urban community." Circulation, vol. 68 , no. 1 , pp. 50-58, jul 1983 .

[2] W. Nichols, M. O'Rourke, and C. Vlachopoulos, "Aging," in McDonald's Blood Flow in Arteries 6th Edition: Theoretical, Experimental and Clinical Principles, R. Reneman, Ed. CRC Press, jul 2011, pp. 411-446.

[3] R. T. Lee, A. J. Grodzinsky, E. H. Frank, R. D. Kamm, and F. J. Schoen, "Structure-dependent dynamic mechanical behavior of fibrous caps from human atherosclerotic plaques." Circulation, vol. 83, no. 5, pp. 1764-1770, may 1991.
[4] W. Nichols, M. O'Rourke, and C. Vlachopoulos, "Hypertension," in McDonald's Blood Flow in Arteries 6th Edition: Theoretical, Experimental and Clinical Principles, R. Reneman, Ed. CRC Press, jul 2011, pp. 411-446.

[5] K. Fujikura, J. Luo, V. Gamarnik, M. Pernot, R. Fukumoto, M. D. Tilson, and E. E. Konofagou, "A novel noninvasive technique for pulse-wave imaging and characterization of clinically-significant vascular mechanical properties in vivo," Ultrasonic Imaging, vol. 29, no. 3, pp. 137-154, jul 2007.

[6] M. Tanter and M. Fink, "Ultrafast imaging in biomedical ultrasound," IEEE Transactions on Ultrasonics, Ferroelectrics, and Frequency Control, vol. 61, no. 1, pp. 102-119, jan 2014.

[7] J. Udesen, F. Gran, K. Hansen, J. Jensen, C. Thomsen, and M. Nielsen, "High frame-rate blood vector velocity imaging using plane waves: Simulations and preliminary experiments," IEEE Transactions on Ultrasonics, Ferroelectrics and Frequency Control, vol. 55, no. 8, pp. 1729-1743, aug 2008.

[8] J. Vappou, J. Luo, and E. E. Konofagou, "Pulse wave imaging for noninvasive and quantitative measurement of arterial stiffness in vivo," American Journal of Hypertension, vol. 23, no. 4, pp. 393-398, apr 2010.

[9] H. Hasegawa and H. Kanai, "Simultaneous imaging of artery-wall strain and blood flow realized by high frame rate acquisition of RF echoes," in 2008 IEEE Ultrasonics Symposium. IEEE, nov 2008.

[10] J. Luo and E. E. Konofagou, "Imaging of wall motion coupled with blood flow velocity in the heart and vessels in vivo: A feasibility study," Ultrasound in Medicine \& Biology, vol. 37, no. 6, pp. 980-995, jun 2011.

[11] I. K. Ekroll, A. Swillens, P. Segers, T. Dahl, H. Torp, and L. Lovstakken, "Simultaneous quantification of flow and tissue velocities based on multi-angle plane wave imaging," IEEE Transactions on Ultrasonics, Ferroelectrics and Frequency Control, vol. 60, no. 4, pp. 727-738, apr 2013.

[12] V. Perrot, L. Petrusca, A. Bernard, D. Vray, and H. Liebgott, "Simultaneous pulse wave and flow estimation at high-framerate using plane wave and transverse oscillation on carotid phantom," in 2017 IEEE International Ultrasonics Symposium (IUS). IEEE, sep 2017.

[13] C. Kasai, K. Namekawa, A. Koyano, and R. Omoto, "Real-time two-dimensional blood flow imaging using an autocorrelation technique," IEEE Transactions on Sonics and Ultrasonics, vol. 32, no. 3, pp. 458-464, may 1985.

[14] T. Loupas, J. Powers, and R. Gill, "An axial velocity estimator for ultrasound blood flow imaging, based on a full evaluation of the doppler equation by means of a two-dimensional autocorrelation approach," IEEE Transactions on Ultrasonics, Ferroelectrics and Frequency Control, vol. 42, no. 4, pp. 672-688, jul 1995.

[15] S. Salles, D. Garcia, B. Bou-Said, F. Savary, A. Serusclat, D. Vray, and H. Liebgott, "Plane wave transverse oscillation (PWTO): An ultra-fast transverse oscillation imaging mode performed in the fourier domain for $2 \mathrm{~d}$ motion estimation of the carotid artery," in 2014 IEEE 11th International Symposium on Biomedical Imaging (ISBI). IEEE, apr 2014.

[16] T. Bulow and G. Sommer, "Hypercomplex signals-a novel extension of the analytic signal to the multidimensional case," IEEE Transactions on Signal Processing, vol. 49, no. 11, pp. 2844-2852, 2001.

[17] E. Hermeling, K. D. Reesink, L. M. Kornmann, R. S. Reneman, and A. P. Hoeks, "The dicrotic notch as alternative time-reference point to measure local pulse wave velocity in the carotid artery by means of ultrasonography," Journal of Hypertension, vol. 27, no. 10, pp. 2028-2035, oct 2009.

[18] C. Huang, Y. Su, H. Zhang, L.-X. Qian, and J. Luo, "Comparison of different pulse waveforms for local pulse wave velocity measurement in healthy and hypertensive common carotid arteries in vivo," Ultrasound in Medicine \& Biology, vol. 42, no. 5, pp. 1111-1123, may 2016. 